\title{
Neutrosophic Set and Neutrosophic Topological Spaces
}

\author{
${ }^{1}$ A.A.Salama, ${ }^{2}$ S.A.Alblowi \\ ${ }^{I}$ Egypt, Port Said University, Faculty of Sciences Department of Mathematics and Computer Science \\ ${ }^{2}$ Department of Mathematics, King Abdulaziz University, Saudi Arabia
}

\begin{abstract}
Neutrosophy has been introduced by Smarandache [7, 8] as a new branch of philosophy. The purpose of this paper is to construct a new set theory called the neutrosophic set. After given the fundamental definitions of neutrosophic set operations, we obtain several properties, and discussed the relationship between neutrosophic sets and others. Finally, we extend the concepts of fuzzy topological space [4], and intuitionistic fuzzy topological space [5, 6] to the case of neutrosophic sets. Possible application to superstrings and $\zeta^{\infty}$ space-time are touched upon.
\end{abstract}

Keywords: Fuzzy topology; fuzzy set; neutrosophic set; neutrosophic topology

\section{Introduction}

The fuzzy set was introduced by Zadeh [9] in 1965, where each element had a degree of membership. The intuitionstic fuzzy set (Ifs for short) on a universe X was introduced by K. Atanassov [1, 2, 3] in 1983 as a generalization of fuzzy set, where besides the degree of membership and the degree of non- membership of each element. After the introduction of the neutrosophic set concept [7, 8]. In recent years neutrosophic algebraic structures have been investigated. Neutrosophy has laid the foundation for a whole family of new mathematical theories generalizing both their classical and fuzzy counterparts, such as a neutrosophic set theory.

\section{Terminologies}

We recollect some relevant basic preliminaries, and in particular, the work of Smarandache in $[7,8]$, and Atanassov in $[1,2,3]$. Smarandache introduced the neutrosophic components T, I, F which represent the membership, indeterminacy, and non-membership values respectively, where $\left|0^{-}, 1^{+}\right|$is nonstandard unit interval.

\subsection{Definition. $[3,4]$}

Let T, I,F be real standard or nonstandard subsets of $\rfloor 0^{-}, 1^{+}\lfloor$, with

Sup_T=t_sup, inf_T=t_inf

Sup_I=i_sup, inf_I=i_inf

Sup_F=f_sup, inf_F=f_inf

$\mathrm{n}$-sup=t_sup+i_sup+f_sup

n-inf $=$ t_inf + i_inf + f_inf,

T, I, F are called neutrosophic components

\section{Neutrosophic Sets and Its Operations} operations.

We shall now consider some possible definitions for basic concepts of the neutrosophic set and its

\subsection{Definition}

Let $X$ be a non-empty fixed set. $A$ neutrosophic set ( $N S$ for short) $A$ is an object having the form $A=\left\{\left\langle x, \mu_{A}(x), \sigma_{A}(x), \gamma_{A}(x)\right\rangle: x \in X\right\}$ Where $\mu_{A}(x), \sigma_{A}(x)$ and $\gamma_{A}(x)$ which represent the degree of member ship function (namely $\mu_{A}(x)$ ), the degree of indeterminacy (namely $\sigma_{A}(x)$ ), and the degree of non-member ship (namely $\gamma_{A}(x)$ ) respectively of each element $x \in X$ to the set $A$.

\subsection{Remark}

A neutrosophic $A=\left\{<x, \mu_{A}(x), \sigma_{A}(x), \gamma_{A}(x)>: x \in X\right\}$ can be identified to an ordered triple $\left\langle\mu_{A}, \sigma_{A}, \gamma_{A}\right\rangle$ in $]^{-0} 0,1^{+}[$on. $X$. 
3.2 Remark

For the sake of simplicity, we shall use the symbol $A=<x, \mu_{A}, \sigma_{A}, \gamma_{A}>$ for the

NS $A=\left\{<x, \mu_{A}(x), \sigma_{A}(x), \gamma_{A}(x)>: x \in X\right\}$

3.1 Example

Every IFS $A$ a non-empty set $X$ is obviously on $N S$ having the form

$A=\left\{<x, \mu_{A}(x), 1-\left(\mu_{A}(x)+\gamma_{A}(x)\right), \gamma_{A}(x)>: x \in X\right\}$

Since our main purpose is to construct the tools for developing neutrosophic set and neutrosophic topology, we must introduce the NSS $0_{N}$ and $1_{N}$ in $X$ as follows:

$0_{N}$ may be defined as:

$\left(0_{1}\right) \quad 0_{N}=\{\langle x, 0,0,1\rangle: x \in X\}$

$\left(0_{2}\right) 0_{N}=\{\langle x, 0,1,1\rangle: x \in X\}$

$\left(0_{3}\right) 0_{N}=\{\langle x, 0,1,0\rangle: x \in X\}$

$\left(0_{4}\right) \quad 0_{N}=\{\langle x, 0,0,0\rangle: x \in X\}$

$1_{N}$ may be defined as:

$\left(1_{1}\right) 1_{N}=\{\langle x, 1,0,0\rangle: x \in X\}$

$\left(1_{2}\right) 1_{N}=\{\langle x, 1,0,1\rangle: x \in X\}$

(1 $\left.1_{3}\right) 1_{N}=\{\langle x, 1,1,0\rangle: x \in X\}$

(1 $\left.1_{4}\right) 1_{N}=\{\langle x, 1,1,1\rangle: x \in X\}$

\subsection{Definition}

Let $A=\left\langle\mu_{A}, \sigma_{A}, \gamma_{A}\right\rangle$ a $N S$ on $X$, then the complement of the set $A(C(A)$, for short ) maybe defined as three kinds of complements
$\left(C_{1}\right) C(A)=\left\{\left\langle x, 1-\mu_{A}(x), 1-\gamma_{A}(x)\right\rangle: x \in X\right\}$,
$\left(C_{2}\right) C(A)=\left\{\left\langle x, \gamma_{A}, \sigma_{A}(x), \mu_{A}(x)\right\rangle: x \in X\right\}$
$\left(C_{3}\right) C(A)=\left\{\left\langle x, \gamma_{A}, 1-\sigma_{A}(x), \mu_{A}(x)\right\rangle: x \in X\right\}$

One can define several relations and operations between NSS follows:

\subsection{Definition}

Let $x$ be a non-empty set, and NSS $A$ and $B$ in the form $A=\left\langle x, \mu_{A}(x), \sigma_{A}(x), \gamma_{A}(x)\right\rangle$, $B=\left\langle x, \mu_{B}(x), \sigma_{B}(x), \gamma_{B}(x)\right\rangle$, then we may consider two possible definitions for subsets $(A \subseteq B)$

$(A \subseteq B)$ may be defined as

(1) $A \subseteq B \Leftrightarrow \mu_{A}(x) \leq \mu_{B}(x), \gamma_{A}(x) \geq \gamma$ and $\sigma_{A}(x) \leq \sigma_{B}(x) \quad \forall x \in X$

(2) $A \subseteq B \Leftrightarrow \mu_{A}(x) \leq \mu_{B}(x), \gamma_{A}(x) \geq \gamma_{B}(x)$ and $\sigma_{A}(x) \geq \sigma_{B}(x)$

\subsection{Proposition}

For any neutrosophic set $A$ the following are holds

(1) $0_{N} \subseteq A, \quad 0_{N} \subseteq 0_{N}$

(2) $A \subseteq 1_{N}, 1_{N} \subseteq 1_{N}$

\subsection{Definition}

Let $X$ be a non-empty set, and $A=<x, \mu_{A}(x), \gamma_{A}(x), \sigma_{A}(x)>, B=<x, \mu_{B}(x), \sigma_{B}(x), \gamma_{B}(x)>$ are NSS. Then

(1) $A \cap B$ maybe defined as:

$\left(I_{1}\right) A \cap B=<x, \mu_{A}(x) \cdot \mu_{B}(x), \sigma_{A}(x) \cdot \sigma_{B}(x)$,

$\gamma_{A}(x) \cdot \gamma_{B}(x)>$

$\left(I_{2}\right) A \cap B=<x, \mu_{A}(x) \wedge \mu_{B}(x), \sigma_{A}(x) \wedge \sigma_{B}(x)$,

$\gamma_{A}(x) \vee \gamma_{B}(x)>$

$\left(I_{3}\right) A \cap B=<x, \mu_{A}(x) \wedge \mu_{B}(x), \sigma_{A}(x) \vee \sigma_{B}(x), \gamma_{A}(x) \vee \gamma_{B}(x)>$ 
(2) $A \cup B$ may be defined as:

$\left(U_{1}\right) \quad A \cup B=<x, \mu_{A}(x) \vee \mu_{B}(x), \sigma_{A}(x) \vee \sigma_{B}(x)$,

$\gamma_{A}(x) \wedge \gamma_{B}(x)>$

$\left(U_{2}\right) \quad A \cup B=<x, \mu_{A}(x) \vee \mu_{B}(x), \sigma_{A}(x) \wedge \sigma_{B}(x)$,

$\gamma_{A}(x) \wedge \gamma_{B}(x)>$

(3) []$A=<x, \mu_{A}(x), \sigma_{A}(x), 1-\mu_{A}(x)>$

(4) $<>A=<x, 1-\gamma_{A}(x), \sigma_{A}(x), \gamma_{A}(x)>$

We can easily generalize the operations of intersection and union in definition 3.4 to arbitrary family of NSS as follow:

\subsection{Definition}

Let $\{A j: j \in J\}$ be a arbitrary family of NSS in $X$, then

(1) $\cap A j$ maybe defined as:

(i) $\cap A j=\left\langle x,{ }_{j \in I} \mu_{A j}(x), \underset{j \in J}{\wedge} \sigma_{A j}(x), \vee \gamma_{A j}(x)\right\rangle$

(ii) $\cap A j=\left\langle x, \wedge \mu_{A j}(x), \vee \sigma_{A j}(x), \vee \gamma_{A j}(x)\right\rangle$

(2) $\cup A j$ maybe defined as:

(i) $\cup A j=\langle x, \vee, \wedge, \wedge\rangle$

(ii) $\bigcup A j=\langle x, \vee, \wedge, \wedge\rangle$

\subsection{Definition}

Let $A$ and $B$ are neutrosophic sets then

$A \mid B$ may be defined as

$A \mid B=\left\langle x, \mu_{A} \wedge \gamma_{B}, \sigma_{A}(x) \sigma_{B}(x), \gamma_{A} \vee \mu_{B}(x)\right\rangle$

\subsection{Proposition}

For all $A, B$ two neutrosophic sets then the following are true

(1) $C(A \cap B)=C(A) \cup C(B)$

(2) $C(A \cup B)=C(A) \cap C(B)$

\section{Neutrosophic Topological Spaces}

Here we extend the concepts of fuzzy topological space [4], and intuitionistic fuzzy topological space [5, 7] to the case of neutrosophic sets.

\subsection{Definition}

A neutrosophic topology ( $N T$ for short) an a non empty set $X$ is a family $\tau$ of neutrosophic subsets in $X$ satisfying the following axioms

$\left(N T_{1}\right) O_{N}, 1_{N} \in \tau$

$\left(N T_{2}\right) G_{1} \cap G_{2} \in \tau$ for any $G_{1}, G_{2} \in \tau$,

$\left(N T_{3}\right) \cup \boldsymbol{G}_{i} \in \tau \quad \forall\left\{\boldsymbol{G}_{i}: \boldsymbol{i} \in \boldsymbol{J}\right\} \subseteq \tau$

In this case the pair $(X, \tau)$ is called a neutrosophic topological space (NTS for short) and any neutrosophic set in $\tau$ is known as neuterosophic open set (NOS for short) in $X$. The elements of $\tau$ are called open neutrosophic sets, A neutrosophic set $\mathrm{F}$ is closed if and only if it $\mathrm{C}(\mathrm{F})$ is neutrosophic open.

\subsection{Example}

Any fuzzy topological space $\left(X, \tau_{0}\right)$ in the sense of Chang is obviously a NTS in the form $\tau=\left\{A: \mu_{A} \in \tau_{0}\right\}$ wherever we identify a fuzzy set in $X$ whose members ship function is $\mu_{A}$ with its counterpart.

4.1. Remark Neutrosophic topological spaces are very natural generalizations of fuzzy topological spaces allow more general functions to be members of fuzzy topology.

\subsection{Example}

Let $X=\{x\}$ and

$A=\{\langle x, 0.5,0.5,0.4\rangle: x \in X\}$

$B=\{\langle x, 0.4,0.6,0.8\rangle: x \in X\}$

$D=\{\langle x, 0.5,0.6,0.4\rangle: x \in X\}$ 
$C=\{\langle x, 0.4,0.5,0.8\rangle: x \in X\}$

Then the family $\tau=\left\{O_{n}, 1_{n}, A, B, C, D\right\}$ of $\mathrm{N} S s$ in $X$ is neutrosophic topology on $X$

\subsection{Example}

Let $\left(X, \tau_{0}\right)$ be a fuzzy topological space in changes sense such that $\tau_{0}$ is not indiscrete suppose now that $\tau_{\mathrm{O}}=\left\{\mathrm{O}_{N}, 1_{N}\right\} \cup\left\{V_{j}: j \in J\right\}$ then we can construct two NTSS on $X$ as follows

a) $\tau_{0}=\left\{\mathrm{O}_{N}, 1_{N}\right\} \cup\left\{<x, V_{j}, \sigma(x), 0>: j \in J\right\}$.

b) $\tau_{0}=\left\{0_{N}, 1_{N}\right\} \cup\left\{<x, V_{j}, 0, \sigma(x), 1-V_{j}>: j \in J\right\}$.

\subsection{Proposition}

Let $(X, \tau)$ be a NTS on $X$, then we can also construct several NTSS on $X$ in the following way:

a) $\tau_{o, 1}=\{[] G: G \in \tau\}$,

b) $\tau_{o, 2}=\{<>G: G \in \tau\}$,

Proof

a) $\left(N T_{1}\right)$ and $\left(N T_{2}\right)$ are easy.

$\left(N T_{3}\right) \operatorname{Let}\left\{\left[G_{j}: j \in J, G_{j} \in \tau\right\} \subseteq \tau_{0,1}\right.$. Since

$\left.\cup G_{j}=\left\{\left(x, \vee \mu_{G_{j}}, \vee \sigma_{G_{j}}, \wedge \gamma_{G_{j}}\right)\right\} \operatorname{or}\left\{\left\{x, \vee \mu_{G_{j}}, \wedge \sigma_{G_{j}}, \wedge \gamma_{G_{j}}\right)\right\} \operatorname{or}\left\{x, \vee \mu_{G_{j}}, \wedge \sigma_{G_{j}}, \vee \gamma_{G_{j}}\right)\right\} \in \tau$, we have

$\cup\left([] G_{j}\right)=\left\{x, \vee \mu_{G_{j}}, \vee \sigma_{G_{j}}, \wedge\left(1-\mu_{G_{j}}\right)\right\}$ or $\left\{x, \vee \mu_{G_{j}}, \vee \sigma_{G_{j}},\left(1-\vee \mu_{G_{j}}\right)\right\} \in \tau_{0,1}$

b) This similar to (a)

\subsection{Definition}

Let $\left(X, \tau_{1}\right),\left(X, \tau_{2}\right)$ be two neutrosophic topological spaces on $X$. Then $\tau_{1}$ is said be contained in $\tau_{2}$ (in symbols $\tau_{1} \subseteq \tau_{2}$ ) if $G \in \tau_{2}$ for each $G \in \tau_{1}$. In this case, we also say that $\tau_{1}$ is coarser than $\tau_{2}$.

\subsection{Proposition}

Let $\left\{\tau_{j}: j \in J\right\}$ be a family of NTSS on $X$. Then $\cap \tau_{j}$ is A neutrosophic topology on $X$.Furthermore, $\cap \tau_{j}$ is the coarsest $N T$ on $X$ containing all. $\tau_{j}, \mathrm{~s}$

Proof. Obvious

\subsection{Definition}

The complement of $A$ (C (A) for short) of NOS. $A$ is called a neutrosophic closed set (NCS for short) in $X$. Now, we define neutrosophic closure and interior operations in neutrosophic topological spaces:

\subsection{Definition}

Let $(X, \tau)$ be NTS and $A=<x, \mu_{A}(x), \gamma_{A}(x), \sigma_{A}(x)>$ be a $N S$ in $X$.

Then the neutrosophic closer and neutrosophic interior of Aare defined by $\operatorname{NCl}(A)=\frown\{K: K$ is an NCS in $\mathrm{X}$ and $\mathrm{A} \subseteq \mathrm{K}\}$

$\operatorname{NInt}(A)=\cup\{G: G$ is an NOS in $\mathrm{X}$ and $\mathrm{G} \subseteq \mathrm{A}\}$.It can be also shown that

It can be also shown that $N C l(A)$ is $N C S$ and $\operatorname{NInt}(A)$ is a $N O S$ in $X$

a) $A$ is in $X$ if and only if $\operatorname{NCl}(A)$.

b) $A$ is $\operatorname{NCS}$ in $X$ if and only if $\operatorname{NInt}(A)=A$.

\subsection{Proposition}

For any neutrosophic set $A$ in $(x, \tau)$ we have

(a) $\operatorname{NCl}(C(A)=C(N \operatorname{Int}(A)$,

(b) $\operatorname{NInt}(C(A))=C(N C l(A))$.

Proof.

a) Let $A=\left\{\left\langle x, \mu_{A}, \sigma_{A}, \nu_{A}>: x \in X\right\}\right.$ and suppose that the family of neutrosophic subsets contained in

$A$ are indexed by the family if NSS contained in $A$ are indexed by the 
family $A=\left\{<x, \mu_{G_{i}}, \sigma_{G_{i}}, v_{G_{i}}>: i \in J\right\}$. Then we see that $\operatorname{NInt}(A)=\left\{<x, \vee \mu_{G_{i}}, \vee \sigma_{G_{i}}, \wedge v_{G_{i}}>\right\}$ and hence $C(\operatorname{NInt}(A))=\left\{<x, \wedge \mu_{G_{i}}, \vee \sigma_{G_{i}}, \vee v_{G_{i}}>\right\}$. Since $C(A)$ and $\mu_{G_{i}} \leq \mu_{A}$ and $v_{G_{i}} \geq v_{A}$ for each $i \in J$, we obtaining $C(A)$. i.e $\operatorname{NCl}(C(A))=\left\{<x, \wedge v_{G_{i}}, \vee \sigma_{G_{i}}, \vee \mu_{G_{i}}>\right\}$. Hence

$\operatorname{NCl}(C(A)=C(\operatorname{NInt}(A)$, follows immediately

b) This is analogous to (a).

\subsection{Proposition}

Let $(x, \tau)$ be a NTS and $A, B$ be two neutrosophic sets in $X$. Then the following properties hold:

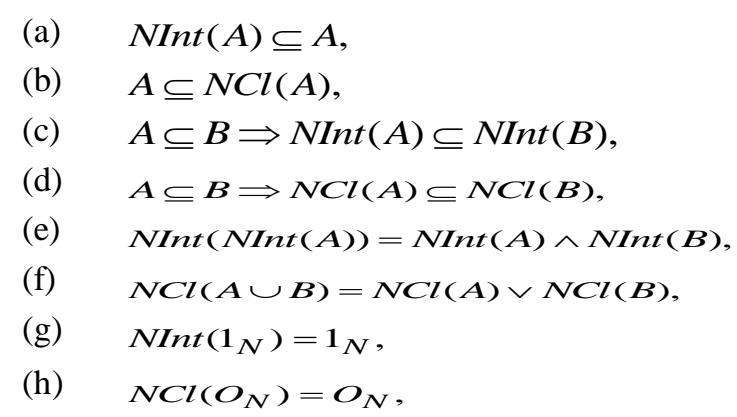

Proof (a), (b) and (e) are obvious (c) follows from (a) and Definitions.

\section{References}

[1] K. Atanassov, intuitionistic fuzzy sets, in V.Sgurev, ed., Vii ITKRS Session, Sofia(June 1983 central Sci. and Techn. Library, Bulg. Academy of Sciences( 1984)).

[2] K. Atanassov, intuitionistic fuzzy sets, Fuzzy Sets and Systems 20(1986)87-96.

[3] K. Atanassov, Review and new result on intuitionistic fuzzy sets , preprint IM-MFAIS-1-88, Sofia, 1988.

[4] C.L. Chang, Fuzzy Topological Spaces, J. Math. Anal. Appl. 24 (1968)182-1 90.

[5] Dogan Coker, An introduction to intuitionistic fuzzy topological spaces, Fuzzy Sets and Systems. 88(1997)81-89.

[6] Reza Saadati, Jin HanPark, On the intuitionistic fuzzy topological space, Chaos, Solitons and Fractals 27(2006)331-344

[7] Florentin Smarandache, Neutrosophy and Neutrosophic Logic, First International Conference on Neutrosophy, Neutrosophic Logic , Set, Probability, and Statistics University of New Mexico, Gallup, NM 87301, USA(2002), smarand@unm.edu

[8] F. Smarandache. A Unifying Field in Logics: Neutrosophic Logic. Neutrosophy, Neutrosophic Set, Neutrosophic Probability. American Research Press, Rehoboth, NM, 1999.

[9] L.A. Zadeh, Fuzzy Sets, Inform and Control 8(1965)338-353 